\title{
Static axisymmetric space-times with prescribed multipole moments
}

\author{
Thomas Bäckdahl*, Magnus Herberthson*
}

\begin{abstract}
In this article we develop a method of finding the static axisymmetric space-time corresponding to any given set of multipole moments. In addition to an implicit algebraic form for the general solution, we also give a power series expression for all finite sets of multipole moments. As conjectured by Geroch we prove in the special case of axisymmetry, that there is a static space-time for any given set of multipole moments subject to a (specified) convergence criterion. We also use this method to confirm a conjecture of Hernández-Pastora and Martín concerning the monopole-quadropole solution.
\end{abstract}

\section{Introduction}

The relativistic multipole moments of vacuum static asymptotically flat spacetimes have been defined by Geroch [5], and this definition has been extended by Hansen [8] to the stationary case. There are other definitions [2], 14, 11] or approaches [3], 10]; for instance, Thorne [14] has suggested an alternative definition which is equivalent if the space-time has nonzero mass 6 .

The recursive definition of Geroch (11) produces a family of totally symmetric and trace-free tensors, which are to be evaluated at a certain point. These values will then provide the moments of the space-time in question. Even in the case of static axisymmetric space-times, where all solutions in a sense are known (see [15] and section 2] below), the actual calculations of the tensors in (11) are non-trivial.

In [7, it was shown how the moments in the axisymmetric case can be obtained through a set of recursively defined real valued functions $\left\{f_{n}\right\}_{n=0}^{\infty}$ on $\mathbb{R}$. The moments are then given by the values $\left\{f_{n}(0)\right\}_{n=0}^{\infty}$. In this way, one can easily calculate 'any' desired number of moments. By exploring the conformal freedom of the construction, it was also shown how all moments could be captured in one real valued function $y$, where the moments appeared as the derivatives of $y$ at 0 .

In this paper this feature will be explored further. Namely, we will ask the question: which static axisymmetric space-time corresponds to a given set of multipole moments? In the case of a finite number of moments, it is possible to explicitly write down the metric for the space-time in terms of a power series. Through this, we can, for instance, prove the monopole-quadropole conjecture

\footnotetext{
*Department of Mathematics, Linköping University, SE-581 83 Linköping, Sweden. e-mail: thbac@mai.liu.se, maher@mai.liu.se
} 
by Hernández-Pastora and Martín, 9]. In the general case, we find an implicit relation for the moment generating function and a function related to the Weyl solutions. Using this relation, we can give the precise condition on the moments in order to have a corresponding physical space-time with these moments. Thus, this proves a conjecture of Geroch [5] in the special case of axisymmetry.

\section{Explicit moments of the axisymmetric Weyl solutions}

We consider a static space-time $M$, with $V$ a 3-surface orthogonal to the timelike Killing vector $\xi^{a}$. It is required that $V$ is asymptotically flat, i.e., if $h_{a b}$ is the positive definite induced metric on $V$, there exists a 3-manifold $\widetilde{V}$ and a conformal factor $\Omega$ satisfying

(i) $\widetilde{V}=V \cup \Lambda$, where $\Lambda$ is a single point

(ii) $\tilde{h}_{a b}=\Omega^{2} h_{a b}$ is a smooth metric on $\widetilde{V}$

(iii) At $\Lambda, \Omega=0, \tilde{D}_{a} \Omega=0, \tilde{D}_{a} \tilde{D}_{b} \Omega=2 \tilde{h}_{a b}$,

where $\tilde{D}_{a}$ is the derivative operator associated with $\tilde{h}_{a b}$.

On $M$, one defines the scalar potential ${ }^{1} \psi=1-\sqrt{-\xi_{a} \xi^{a}}$. The multipole moments of $M$ are then defined on $\widetilde{V}$ as certain derivatives of the scalar potential $\tilde{\psi}=\psi / \sqrt{\Omega}$ at $\Lambda$. More explicitly, following [5], let $\widetilde{R}_{a b}$ denote the Ricci tensor of $\widetilde{V}$, and let $P=\tilde{\psi}$. Define the sequence $P, P_{a_{1}}, P_{a_{1} a_{2}}, \ldots$ of tensors recursively:

$$
P_{a_{1} \ldots a_{n}}=C\left[\tilde{D}_{a_{1}} P_{a_{2} \ldots a_{n}}-\frac{(n-1)(2 n-3)}{2} \widetilde{R}_{a_{1} a_{2}} P_{a_{3} \ldots a_{n}}\right],
$$

where $C[\cdot]$ stands for taking the totally symmetric and trace-free part. The multipole moments of $M$ are then defined as the tensors $P_{a_{1} \ldots a_{n}}$ at $\Lambda$.

If, in addition to the requirement that $M$ is static and asymptotically flat, we also impose the condition that $M$ is axisymmetric, all solutions are in principle known as corresponding to solutions of the Laplace equation in flat 3-space [15]. The metric can then be written

$$
d s^{2}=-e^{2 \alpha} d t^{2}+e^{2(\beta-\alpha)}\left(d R^{2}+d Z^{2}\right)+R^{2} e^{-2 \alpha} d \phi^{2},
$$

where $\alpha=\frac{1}{2} \ln \left|\xi_{a} \xi^{a}\right|$, which vanishes at infinity, is axisymmetric and flatharmonic with respect to the cylindrical coordinates $R, Z$ and $\phi$. Furthermore, $\beta$ is the solution to $\partial_{R} \beta=R\left[\left(\partial_{R} \alpha\right)^{2}-\left(\partial_{Z} \alpha\right)^{2}\right], \partial_{Z} \beta=2 R\left(\partial_{R} \alpha\right)\left(\partial_{Z} \alpha\right), \partial_{\phi} \beta=0$ which vanish at infinity. The metric on $V$ is $d s^{2}=e^{2(\beta-\alpha)}\left(d R^{2}+d Z^{2}\right)+$ $R^{2} e^{-2 \alpha} d \phi^{2}$, and to conformally compactify $V$ and introduce suitable coordinates on $\widetilde{V}$, we let, [5], [7], $\rho=\frac{R}{R^{2}+Z^{2}}, z=\frac{Z}{R^{2}+Z^{2}}$ and define the spherical coordinates $r, \theta, \phi$ via $\rho=r \sin \theta, z=r \cos \theta, \phi=\phi$. Choosing as conformal factor $\Omega=e^{\alpha-\beta} /\left(R^{2}+Z^{2}\right)$, the metric for $\widetilde{V}$ in a neighbourhood of $\Lambda$ (which is now the origin point) is

$$
d \tilde{s}^{2}=e^{-2 \beta} r^{2} \sin ^{2} \theta d \phi^{2}+\left(d r^{2}+r^{2} d \theta^{2}\right)
$$

\footnotetext{
${ }^{1}$ Note that we have changed sign compared to [5]. This gives the monopole $m$ rather than $-m$ for the Schwarzschild solution.
} 
In terms of the spherical coordinates $r, \theta, \phi$, the function ${ }^{2} \widetilde{\alpha}=-\alpha / r$ is harmonic and $\beta$ satisfies $\left(r \partial_{r}-i \partial_{\theta}\right) \beta=i \sin \theta e^{i \theta}\left[\left(r \partial_{r}-i \partial_{\theta}\right) \alpha\right]^{2}$. The conformal factor $\Omega$ is not uniquely determined. One can make a further conformal transformation of $\widetilde{V}$, using as conformal factor $e^{\kappa}$, where $\kappa$ is any smooth function on $\widetilde{V}$ with $\kappa(\Lambda)=0$. Thus $\kappa$ reflects the freedom in choosing $\Omega$. Of particular importance is the value of $\kappa^{\prime}(0)$. Namely, under a change $\Omega \rightarrow \Omega e^{\kappa}$, a non-zero $\kappa^{\prime}(0)$ changes the moments defined by (11) in a way which corresponds to a 'translation' of the physical space [5]. The potential $P$ is

$$
P=\tilde{\psi}=\psi / \sqrt{\Omega}=\frac{e^{(\beta-\kappa) / 2}}{r}\left(e^{-\alpha / 2}-e^{\alpha / 2}\right) .
$$

For the multiple moments $P_{a_{1} \ldots a_{n}}(\Lambda)$, the axisymmetry implies that [5] at $\Lambda$, $P_{a_{1} \ldots a_{n}}$ is proportional to $C\left[z_{a_{1}} z_{a_{2}} \cdots z_{a_{n}}\right]$, where $z_{a}=(d z)_{a}$, so that

$$
P_{a_{1} \ldots a_{n}}(\Lambda)=m_{n} C\left[z_{a_{1}} z_{a_{2}} \cdots z_{a_{n}}\right], n \geq 1, \quad m_{0}=P(\Lambda) .
$$

Thus, in the Weyl case, the moments are given by the sequence $\left(m_{0}, m_{1}, m_{2}, \ldots\right)$. As shown in [7], all these moments can be collected into one single function $y: \mathbb{R}^{+} \cup 0 \rightarrow \mathbb{R}$, where the moments appear as derivatives of $y$ at 0 .

This is possible due to the form of $\tilde{\alpha}$ ( $P_{n}$ being the Legendre polynomials)

$$
\tilde{\alpha}(r, \theta)=\sum_{n=0}^{\infty} a_{n} r^{n} P_{n}(\cos \theta)
$$

together with the form of $C\left[z_{a_{1}} z_{a_{2}} \cdots z_{a_{n}}\right]$ at $\Lambda$. We can also write $\beta(r, \theta)$ explicitly as $\beta=\sum_{k=0}^{\infty} b_{k}(\cos \theta) r^{k+2}$, with, c.f. [9],

$$
b_{k}(z)=\sum_{l=0}^{k} a_{l} a_{k-l} \frac{(l+1)(k-l+1)}{k+2}\left[P_{l+1}(z) P_{k-l+1}(z)-P_{l}(z) P_{k-l}(z)\right] .
$$

As explained in [7], to determine the moments $m_{n}$, it is sufficient to follow the leading order terms of $P_{n}$ in the expansion (2). The leading term of $P_{n}$ is ${ }^{3}$ $\frac{(2 n) !}{2^{n} n !^{2}}=\frac{2^{n}\left(\frac{1}{2}\right)_{n}}{n !}$. Therefore, in conjunction with (2), we also define

$$
Y(r)=\sum_{n=0}^{\infty} a_{n} r^{n} \frac{(2 n) !}{2^{n} n !^{2}}
$$

We can now state the following theorem, taken from [7],

Theorem 1 Suppose that a static axisymmetric asymptotically flat space-time $M$ is given by the flat-harmonic function $\alpha$, which after conformal rescaling is given by (2). Let $Y$ be given by (6), put $\beta=\left(Y+2 r \frac{d Y}{d r}\right)^{2}, h(r)=\int_{0}^{r} 2 r \beta(r) d r$ and define $\kappa$ through

$$
\kappa(r)=-\ln \left(-r \int \frac{e^{h(r)}}{r^{2}} d r\right) .
$$

Put $\rho(r)=r e^{\kappa(r)}$ and define $y: \mathbb{R}^{+} \cup 0 \rightarrow \mathbb{R}$ implicitly by $y(\rho)=e^{-\kappa(r) / 2} Y(r)$. Then the multipole moments $m_{0}, m_{1}, m_{2}, \ldots$ of $M$ are given by $m_{n}=\frac{d^{n} y}{d \rho^{n}}(0)$.

In the definition of $\kappa$, there appears a constant of integration. This constant affects $\kappa^{\prime}(0)$. In particular, one can make $\kappa^{\prime}(0)=0$.

\footnotetext{
${ }^{2}$ With this choice of sign, the Schwarzschild solution will also have positive Weyl moments.

${ }^{3}(a)_{n}=a(a+1)(a+2) \ldots(a+n-1)=\Gamma(a+n) / \Gamma(a)$
} 


\subsection{The Schwarzschild solution}

In this section we illustrate how Theorem 1 can be used to calculate the moments of the Schwarzschild solution. As is well known, the Weyl monopole, i.e., $\alpha \equiv 1$ does not correspond to the Schwarzschild solution. Rather, for Schwarzschild, the corresponding Weyl potential is

$$
\tilde{\alpha}(r, \theta)=\frac{1}{2 r} \ln \frac{\sqrt{1+2 r m \cos \theta+r^{2} m^{2}}+\cos \theta+r m}{\sqrt{1-2 r m \cos \theta+r^{2} m^{2}}+\cos \theta-r m}
$$

so that $\tilde{\alpha}(r, 0)=\sum_{n=0}^{\infty} a_{n} r^{n}=\frac{1}{2 r} \ln \frac{1+m r}{1-m r}=\sum_{n \geq 0 \text {, odd }}^{\infty} \frac{m^{n}}{n} r^{n-1}$ and consequently $Y(r)=\frac{1}{r} \sum_{n \geq 0}^{\infty}$, odd $\frac{m^{n}}{n} r^{n} \frac{(2 n-2) !}{2^{n-1}(n-1) !^{2}}=\frac{m \sqrt{2}}{\sqrt{1+\sqrt{1-4(m r)^{2}}}}$. One can now follow the steps in Theorem 1 and get $\beta(r)=\frac{\sqrt{1-4 m^{2} r^{2}}-1}{2 r^{2}\left(4 m^{2} r^{2}-1\right)}, h(r)=\ln \left(\frac{1+\sqrt{1-4 m^{2} r^{2}}}{2 \sqrt{1-4 m^{2} r^{2}}}\right)$, $\kappa(r)=-\ln \left(\frac{1}{2}+\frac{\sqrt{1-4 m^{2} r^{2}}}{2}-r \kappa^{\prime}(0)\right), \rho(r)=\frac{2 r}{1-2 \kappa^{\prime}(0)+\sqrt{1-4 m^{2} r^{2}}}$, $r(\rho)=\frac{\rho\left(\rho \kappa^{\prime}(0)+1\right)}{m^{2} \rho^{2}+\left(\rho \kappa^{\prime}(0)+1\right)^{2}}$, and finally

$$
y(\rho)=\frac{m}{\sqrt{\rho \kappa^{\prime}(0)+1}} .
$$

With $\kappa^{\prime}(0)=0$ in ()ㅡ, so that $y(\rho) \equiv m$, it is evident that we have the Schwarzschild solution. This corresponds to an expansion 'around the centre of mass' (where the dipole moment vanishes). A non-zero $\kappa^{\prime}(0)$ gives a 'translated expansion' as explained in [5].

If we just want to calculate any finite number of moments, it is usually easier to use some of the other algorithms from [7]. The strength of Theorem [1 lies in the fact that all moments are handled simultaneously. This will be used in the next section where we will address the question of which $\alpha$ corresponds to a given set of multipole moments.

\section{General multipole}

In this section, we will study the following question. Given a sequence of multipole moments, together with some convergence criteria, what is the corresponding space-time? The moments $\left\{m_{n}\right\}$ are given through the function

$$
y=y(\rho)=\sum_{n=0}^{\infty} \frac{m_{n} \rho^{n}}{n !}
$$

as in Theorem 1] i.e., through its derivatives at 0 . We assume that the series converges in some neighbourhood of $\rho=0$. The space-time is then determined if we know $Y(r)$, since this gives $\alpha$.

We first note from Theorem 1 that $\rho=r e^{\kappa(r)}$. This relates $Y$ and $y$ since $Y=e^{\kappa / 2} y(\rho)=e^{\kappa / 2} y\left(r e^{\kappa}\right)$. However, this relation is somewhat implicit, and therefore of limited use.

Instead, we write $Y=\sqrt{\frac{\rho}{r}} y(\rho)$ and look for a more direct relation between $\rho$ and $r$ : 
Lemma 2 If $y$ is given by (3) and $\rho, \kappa$ are given implicitly by the relations in Theorem 1, then

$$
0=-\frac{\rho}{r}+\rho \int_{0}^{\rho} \frac{1}{\sigma^{2}} \int_{0}^{\sigma} 2 \rho\left(y+2 \rho y_{\rho}\right)^{2} d \rho d \sigma+1+\kappa^{\prime}(0) \rho .
$$

Proof. From Theorem 1 we have that $r / \rho=e^{-\kappa}=-r \int \frac{e^{h(r)}}{r^{2}} d r$ so that $e^{h(r)}=r^{2} \rho_{r} / \rho^{2}$ and $h(r)=\int_{0}^{r} 2 r \beta(r) d r=2 \ln r-2 \ln \rho+\ln \rho_{r}$ and consequently

$$
2 r \beta=\frac{2}{r}-\frac{2 \rho_{r}}{\rho}+\frac{\rho_{r r}}{\rho_{r}},
$$

where $\beta=\left(Y+2 r Y_{r}\right)^{2}$. Putting $G=\sqrt{r} Y(r)=\sqrt{\rho} y(\rho)$, we get

$$
G_{r}^{2}=\frac{1}{4 r}\left(Y(r)+2 r Y_{r}\right)^{2}=\frac{\beta}{4 r} \quad \text { and also } \quad G_{r}^{2}=\frac{1}{4 \rho}\left(y(\rho)+2 \rho y_{\rho}\right)^{2} \rho_{r}^{2},
$$

where the second expression follows from the chain rule, $G_{r}=G_{\rho} \rho_{r}$. Eliminating $\beta$ and $G_{r}$ in (10) and (11), we get $\frac{2 \rho^{2}}{r^{3} \rho_{r}^{2}}-\frac{2 \rho}{r^{2} \rho_{r}}+\frac{\rho^{2} \rho_{r r}}{r^{2} \rho_{r}^{3}}=2 \rho\left(y+2 \rho y_{\rho}\right)^{2}$. However, the left hand side is just $-\frac{d}{d \rho}\left(\frac{\rho^{2}}{r^{2} \rho_{r}}\right)$, so that $\frac{\rho^{2}}{r^{2} \rho_{r}}=-\int_{0}^{\rho} 2 \rho\left(y+2 \rho y_{\rho}\right)^{2} d \rho+$ $C_{1}$. From $\rho=r e^{\kappa(r)}, \kappa(0)=0$, the limit $r \rightarrow 0$ gives $C_{1}=1$. Thus,

$$
\frac{1}{r^{2} \rho_{r}}=-\frac{d}{d \rho}\left(\frac{1}{r}\right)=-\frac{1}{\rho^{2}} \int_{0}^{\rho} 2 \rho\left(y+2 \rho y_{\rho}\right)^{2} d \rho+\frac{1}{\rho^{2}} .
$$

A further integration of (12) followed by a multiplication with $\rho$ yields

$$
0=-\frac{\rho}{r}+\rho \int_{0}^{\rho} \frac{1}{\sigma^{2}} \int_{0}^{\sigma} 2 \rho\left(y+2 \rho y_{\rho}\right)^{2} d \rho d \sigma+1+C_{2} \rho .
$$

By differentiating with respect to $r$, observing that

$$
\rho \frac{d}{d r} \int_{0}^{\rho} \frac{1}{\sigma^{2}} \int_{0}^{\sigma} 2 \rho\left(y+2 \rho y_{\rho}\right)^{2} d \rho d \sigma=\frac{1+r \kappa^{\prime}-e^{\kappa}}{r}
$$

and taking the limit $r \rightarrow 0$, we find that $C_{2}=\kappa^{\prime}(0)$ which is usually, but not always, put to 0 . This gives Lemma 2

We will use this relation extensively in the sequel. For instance, we notice that for a space-time with only a finite number of non-zero moments, $y$ will be a polynomial in $\rho$. This means that (9) will be an algebraic equation which can be solved by the techniques presented in section 4 . For instance, the Schwarzschild solution is given by the constant function $y(\rho)=m$, so that (9), with $\kappa^{\prime}(0)=0$ gives

$$
\rho^{2} m^{2}-\frac{\rho}{r}+1=0,
$$

i.e., $\rho=\frac{2 r}{1+\sqrt{1-4 m^{2} r^{2}}}, Y=\sqrt{\frac{\rho}{r}} y=\frac{\sqrt{2} m}{\sqrt{1+\sqrt{1-4 m^{2} r^{2}}}}$ from which (cf. section 2.1)

$$
\tilde{\alpha}(r, 0)=\frac{1}{2 r} \ln \frac{1+m r}{1-m r} \text {. }
$$




\subsection{On a multipole conjecture due to Geroch}

In [5], it was conjectured that

Two static solutions of Einstein's equations having identical multipole moments coincide, at least in some neighbourhood of $\Lambda$.

and that

Given any set of multipole moments, subject to the appropriate convergence condition, there exists a static solution of Einstein's equations having precisely those moments.

The first conjecture was proven in [1], at least for static space-times with nonzero mass. We will prove the second conjecture for the special case of axisymmetric space-times and provide the precise condition on the moments.

Theorem 3 Given any set of multipole moments $\left\{m_{n}\right\}_{n=0}^{\infty}$ defined by (5), such that

$$
y(\rho)=\sum_{n=0}^{\infty} \frac{m_{n}}{n !} \rho^{n}
$$

converges in some neighbourhood of $\rho=0$, there exists an axisymmetric static solution of Einstein's equations having precisely those moments. Conversely, to every axisymmetric space-time given by $\widetilde{\alpha}(r, 0)$ in (2) with the corresponding set of moments $\left\{m_{n}\right\}_{n=0}^{\infty}$, the right hand side of (13) converges in a neighbourhood of $\rho=0$.

Proof. Suppose $\left\{m_{n}\right\}_{n=0}^{\infty}$ is such that $y(\rho)=\sum_{n=0}^{\infty} \frac{m_{n}}{n !} \rho^{n}$ converges in some neighbourhood of $\rho=0$. From (9) we find that $r(\rho)=\rho /(1+\rho f(\rho))$, where $f(\rho)=\int_{0}^{\rho} \frac{1}{\sigma^{2}} \int_{0}^{\sigma} 2 \rho\left(y+2 \rho y_{\rho}\right)^{2} d \rho d \sigma+\kappa^{\prime}(0)$ is analytic in a neighbourhood of $\rho=$ 0 . From $r(0)=0, r^{\prime}(0) \neq 0$, the inverse function theorem for analytic functions 12 gives that $\rho=\rho(r)$ is also an analytic function, with $\rho(0)=0$, in some neighbourhood of $r=0$. Thus $Y(r)=\sqrt{\rho / r} y(\rho)=\sqrt{1+\rho(r) f(\rho(r))} y(\rho(r))$ is analytic in some neighbourhood of $r=0$. The same condition will then hold for $\widetilde{\alpha}(r, 0)$, so that $\widetilde{\alpha}(r, \theta)$ is (real) analytic in a neighbourhood of $r=0$.

Conversely, suppose $\widetilde{\alpha}(r, \theta)$ is flat-harmonic in a neighbourhood of $r=0$. Then $Y$, given by (6), will also be analytical in a neighbourhood of $r=0$ and from Theorem [1 it is evident that also $\beta$ and $h$ will have this property. Note that $h(0)=h^{\prime}(0)=0$, which implies that $\kappa$ is also analytic in a neighbourhood of $r=0$ and satisfies $\kappa(0)=0$. From $\rho=r e^{\kappa(r)}$, we have $\rho(0)=0, \rho^{\prime}(0) \neq 0$ and that consequently $r$ is an analytic function of $\rho$ near $\rho=0$ with $r(0)=0$. This implies that $y(\rho)=e^{-\kappa(r(\rho)) / 2} Y(r(\rho))$ is analytic in a neighbourhood of $\rho=0$, so that $y(\rho)=\sum_{n=0}^{\infty} c_{n} \rho^{n}$ converges there. From $m_{n}=\frac{d^{n} y}{d \rho^{n}}(0)=c_{n} n$ ! the statement follows.

\subsection{Finite number of moments}

In the special case of a finite number of (non-zero) moments, the function $y$ will be a polynomial in $\rho$. This implies that Lemma 2 will give an algebraic relation between $\rho$ and $r$. Regarding $\rho$ as the unknown, we will have a polynomial equation where the coefficients depends on the desired moments and $r$. Namely, 
suppose $y(\rho)=\sum_{k=0}^{k=n} \frac{m_{k}}{k !} \rho^{k}$ so that all moments $m_{n+1}, m_{n+2}, \ldots$ are zero. From (9) we find that $\rho$ satisfies the equation

$$
a_{2 n} \rho^{2 n}+a_{2 n-1} \rho^{2 n-1}+\cdots+a_{2} \rho^{2}+\left(\kappa^{\prime}(0)-\frac{1}{r}\right) \rho+1=0
$$

so that $a_{0}=1, a_{1}=\kappa^{\prime}(0)-\frac{1}{r}$. All other coefficients $a_{2}, a_{3}, \ldots a_{2 n}$ are polynomial expressions in the moments $m_{0}, m_{1}, \ldots m_{n}$. As we will see in section 4 this equation can be solved in terms of a power series, and this will enable us to write down $Y$ and $\alpha$ explicitly in terms of a power series.

\section{Solving algebraic equations}

If we have a finite number of moments, equation (9) reduces to the algebraic equation (14). In this section we therefore study solutions to a general algebraic equation

$$
a_{n} X^{n}+a_{n-1} X^{n-1}+\cdots+a_{1} X+a_{0}=0 .
$$

This equation can be solved in terms of $\mathcal{A}$-hypergeometric series in the sense of Gel'fand, Kapranov and Zelevinsky, 4]. Sturmfels [13] constructs the solutions explicitly in the form of power series. Loosely speaking, each of the $n$ solutions to (15) can be written down as power series in several ways, depending on how one combines the coefficients $a_{k}$. For our purpose, we seek the solution $\rho$ to (14) where $\rho / r \rightarrow 1$ as $r \rightarrow 0$. Furthermore, since we are looking for solutions which converge near $r=0$, we note that in (14), $a_{1}$ is 'large'.

To be consistent with 13 we introduce the following notations for $u \in \mathbb{Q}$, $v \in \mathbb{Z}$

$$
\begin{gathered}
\mathcal{A}=\left(\begin{array}{lllll}
0 & 1 & \ldots & n-1 & n \\
1 & 1 & \ldots & 1 & 1
\end{array}\right) \\
\gamma(u, v)= \begin{cases}1 & v=0 \\
u(u-1) \ldots(u+v+1) & v<0 \\
0 & 0>u \geq-v, u \in \mathbb{Z} \\
\frac{1}{(u+1)(u+2) \ldots(u+v)} & \text { otherwise }\end{cases}
\end{gathered}
$$

Note that if $u$ is not a negative integer then $\gamma(u, v)=\Gamma(u+1) / \Gamma(u+v+1)$. We also define the formal power series

$$
\left[a_{0}^{u_{0}} a_{1}^{u_{1}} \ldots a_{n}^{u_{n}}\right]=\sum_{\left(v_{0}, \ldots, v_{n}\right) \in \mathcal{L}} \prod_{i=0}^{n}\left(\gamma\left(u_{i}, v_{i}\right) a_{i}^{u_{i}+v_{i}}\right)
$$

where $\mathcal{L}$ is the integer kernel of $\mathcal{A}$, i.e., integer solutions to the equation $\mathcal{A} \bar{v}=0$. Sturmfels [13] then states that $-\left[a_{0} a_{1}^{-1}\right]$ is a solution to (15):

$$
X=-\left[a_{0} a_{1}^{-1}\right]=-\sum_{v_{2}, \ldots, v_{n} \geq 0} a_{0}^{1+v_{0}} a_{1}^{-1+v_{1}} \frac{\gamma\left(-1, v_{1}\right)}{\Gamma\left(2+v_{0}\right)} \prod_{j=2}^{n} \frac{a_{j}^{v_{j}}}{v_{j} !}
$$

where $v_{1}=-\sum_{i=2}^{n} i v_{i}, v_{0}=\sum_{i=2}^{n}(i-1) v_{i}$. We also note that for negative integers $v_{1}, \gamma\left(-1, v_{1}\right)=\prod_{i=0}^{-v_{1}-1}(-1-i)=(-1)^{v_{1}} \Gamma\left(1-v_{1}\right)$, which leads to the 
following expression for the solution

$$
X=\left(\frac{-a_{0}}{a_{1}}\right) \sum_{v_{2} \ldots v_{n} \geq 0} \frac{a_{0}^{\sum_{j=2}^{n}(j-1) v_{j}}\left(\sum_{j=2}^{n} j v_{j}\right) !}{\left(-a_{1}\right)^{\sum_{j=2}^{n} j v_{j}}\left(1+\sum_{j=2}^{n}(j-1) v_{j}\right) !} \prod_{j=2}^{n} \frac{a_{j}^{v_{j}}}{v_{j} !}
$$

Despite its appearance, (16) is useful, since an application to (14), with $\kappa^{\prime}(0)=$ 0 , yields $\rho$ as a power series in terms of $r$. The issue of convergence in (16) will be addressed in section (5).

\section{Powers of the root}

With $\rho$ known in terms of $r$, we can almost write down the function $Y=$ $\sqrt{\rho / r} y(\rho)$ where $y$ is a polynomial. However, we need to take fractional powers of $\rho$. Powers of multiple series are cumbersome, but in this case they can be expressed explicitly by the following extension of the work in [13. We begin with a lemma describing fractional powers of (16) in terms of formal power series. We then extend this to real (positive) powers and prove convergence.

Lemma 4 Let $X$ be the root of (15) given by (16) and assume that $\gamma \in \mathbb{Q}^{+} \backslash \mathbb{Z}$. Then

$$
X^{\gamma}=\left(\frac{-a_{0}}{a_{1}}\right)^{\gamma} \sum_{v_{2} \ldots v_{n} \geq 0} \frac{\gamma a_{0}^{\sum_{j=2}^{n}(j-1) v_{j}} \Gamma\left(\gamma+\sum_{j=2}^{n} j v_{j}\right)}{\left(-a_{1}\right)^{\sum_{j=2}^{n} j v_{j}} \Gamma\left(1+\gamma+\sum_{j=2}^{n}(j-1) v_{j}\right)} \prod_{j=2}^{n} \frac{a_{j}^{v_{j}}}{v_{j} !} .
$$

Proof. We first see [13] that a solution $X$ to (15) satisfies

$$
\begin{aligned}
& \sum_{i=0}^{n} i a_{i} \frac{\partial X}{\partial a_{i}}=-X, \quad \sum_{i=0}^{n} a_{i} \frac{\partial X}{\partial a_{i}}=0, \\
& \frac{\partial^{2} X}{\partial a_{i} \partial a_{j}}=\frac{\partial^{2} X}{\partial a_{k} \partial a_{l}} \quad \text { whenever } \quad i+j=k+l .
\end{aligned}
$$

This implies (c.f. the proof for (18) in [13]) that $Y=X^{\gamma}$ satisfies the following system of equations

$$
\begin{aligned}
& \sum_{i=0}^{n} i a_{i} \frac{\partial Y}{\partial a_{i}}=-\gamma Y, \quad \sum_{i=0}^{n} a_{i} \frac{\partial Y}{\partial a_{i}}=0, \\
& \frac{\partial^{2} Y}{\partial a_{i} \partial a_{j}}=\frac{\partial^{2} Y}{\partial a_{k} \partial a_{l}} \quad \text { whenever } \quad i+j=k+l .
\end{aligned}
$$

The assumption $\gamma \in \mathbb{Q}^{+} \backslash \mathbb{Z}$ together with Lemma 3.1 in Sturmfels 13 implies that $\left[a_{0}^{\gamma} a_{1}^{-\gamma}\right]$ satisfies the system (19), and homogeneity implies that $\tilde{X}=$ $(-1)^{\gamma}\left[a_{0}^{\gamma} a_{1}^{-\gamma}\right]$ also satisfies the same system. Written out explicitly this means that

$$
\tilde{X}=(-1)^{\gamma} \sum_{v_{2}, \ldots, v_{n} \geq 0} a_{0}^{\gamma+v_{0}} a_{1}^{-\gamma+v_{1}} \frac{\Gamma(\gamma+1) \Gamma(1-\gamma)}{\Gamma\left(\gamma+v_{0}+1\right) \Gamma\left(1-\gamma+v_{1}\right)} \prod_{j=2}^{n} \frac{a_{j}^{v_{j}}}{v_{j} !} .
$$

solves (19). In the general case, the occurrence of the fractional power $\gamma$ means that some care has to be taken when choosing branches. In our case however, all 
coefficient $a_{i}$ are real, and in particular $\left(-a_{0} / a_{1}\right)$ is positive if $r$ is small enough. Thus, no resulting ambiguity will occur from $\left(-a_{0} / a_{1}\right)^{\gamma}$. Using the relations $\Gamma\left(1-\gamma+v_{1}\right)=\frac{\pi}{\sin \left(\gamma \pi-v_{1} \pi\right) \Gamma\left(\gamma-v_{1}\right)}$ and $\Gamma(1-\gamma)=\frac{\pi}{\sin (\gamma \pi) \Gamma(\gamma)}$, we write

$$
\tilde{X}=(-1)^{\gamma} \sum_{v_{2}, \ldots, v_{n} \geq 0} a_{0}^{\gamma+v_{0}} a_{1}^{-\gamma+v_{1}} \frac{\gamma \sin \left(\gamma \pi-v_{1} \pi\right) \Gamma\left(\gamma-v_{1}\right)}{\sin (\gamma \pi) \Gamma\left(1+\gamma+v_{0}\right)} \prod_{j=2}^{n} \frac{a_{j}^{v_{j}}}{v_{j} !} .
$$

Plugging in $v_{1}=-\sum_{i=2}^{n} i v_{i}$ and $v_{0}=\sum_{i=2}^{n}(i-1) v_{i}$, we see that $\tilde{X}$ has the form (17). Thus Lemma 4 is proven if we show that $\tilde{X}=X^{\gamma}$.

This will follow from Lemma 5 which says that the solution to (19) of the form (20) is unique provided certain initial values are given. Both $\tilde{X}$ and $X^{\gamma}$ have the form in Lemma 5 below with $b_{0}=1$ and $b_{1}=0$. Therefore, $\tilde{X}=X^{\gamma}$ for all $\gamma \in \mathbb{Q}^{+} \backslash \mathbb{Z}$, and the proof is complete.

Lemma 5 Assume that a solution to the system (19) has the form

$$
Y=\left(-\frac{a_{0}}{a_{1}}\right)^{\gamma} \sum_{j=0}^{\infty} b_{j}\left(a_{0}, a_{2}, a_{3}, \ldots, a_{n}\right)\left(-a_{1}\right)^{-j}
$$

where $b_{j}$ are analytic and that $\gamma$ is not a negative integer. Then $Y$ is determined by the values of $b_{0}$ and $b_{1}$.

Proof. By linearity we can assume $b_{0}=b_{1}=0$ and show that $Y=0$. Now, let $c_{j}\left(a_{0}, a_{2}, \ldots, a_{n}\right)=a_{0}^{\gamma} b_{j}\left(a_{0}, a_{2}, \ldots, a_{n}\right)$. Given $c_{0}=c_{1}=0$, we will prove by induction that $c_{j}=0$ for all $j \geq 0$. Assume that $c_{j-2}=c_{j-1}=0$. $\frac{\partial^{2} Y}{\partial a_{1}^{2}}=\frac{\partial^{2} Y}{\partial a_{0} \partial a_{2}}$ gives $\sum_{k=0}^{\infty}(k+\gamma)(k+\gamma+1)\left(-a_{1}\right)^{-k-\gamma-2} c_{k}=\sum_{k=0}^{\infty} \frac{\partial^{2} c_{k}}{\partial a_{0} \partial a_{2}}$. Identification of the coefficients implies $\frac{\partial^{2} c_{j}}{\partial a_{0} \partial a_{2}}=(j-2+\gamma)(j-1+\gamma) c_{j-2}=0$. The analyticity of $b_{j}$ lets us define $d_{j, k}\left(a_{2}, a_{3}, \ldots, a_{n}\right)$ such that $b_{j}=\sum_{k=0}^{\infty} d_{j, k} a_{0}^{k}$. We can then write $0=\frac{\partial^{2} c_{j}}{\partial a_{0} \partial a_{2}}=\sum_{k=0}^{\infty}(k+\gamma) a_{0}^{k+\gamma-1} \frac{\partial d_{j, k}}{\partial a_{2}}$. Identification of the coefficients implies $\frac{\partial d_{j, k}}{\partial a_{2}}=0$. Hence, $d_{j, k}\left(a_{2}, a_{3}, \ldots, a_{n}\right)=d_{j, k}\left(a_{3}, \ldots, a_{n}\right)$ for all $k$. From (19) we also have $\frac{\partial^{2} Y}{\partial a_{0} \partial a_{m}}=\frac{\partial^{2} Y}{\partial a_{1} \partial a_{m-1}}, \quad 2<m \leq n$, which gives

$$
\frac{\partial^{2} c_{j}}{\partial a_{0} \partial a_{m}}=(j-1+\gamma) \frac{\partial c_{j-1}}{\partial a_{m-1}}=0, \quad 2<m \leq n .
$$

Using this in the same way as above gives that $d_{j, k}$ does not depend on $a_{m}$, $m=3,4, \ldots n$. Therefore each $d_{j, k}$ is constant. Thus,

$$
Y=\left(-\frac{a_{0}}{a_{1}}\right)^{\gamma}\left[b_{j}\left(a_{0}\right)\left(-a_{1}\right)^{-j}+\sum_{k=j+1}^{\infty} b_{k}\left(a_{0}, a_{2}, a_{3}, \ldots, a_{n}\right)\left(-a_{1}\right)^{-k}\right] .
$$

However, using $-\gamma Y=\sum_{i=0}^{n} i a_{i} \frac{\partial Y}{\partial a_{i}}$ from (19) and looking at the coefficient of $\left(-a_{1}\right)^{-j-\gamma}$, we see that $-\gamma a_{0}^{\gamma} b_{j}\left(a_{0}\right)=-(j+\gamma) a_{0}^{\gamma} b_{j}\left(a_{0}\right)$, so that $b_{j}$ and therefore $c_{j}$ are zero. Induction gives $c_{j}=0$ for all $j \in \mathbb{N}$. Hence the lemma is proven.

Theorem 6 Let $X$ be the root of (15) given by (16) and assume that $\gamma \in \mathbb{R}^{+}$. Then (17) holds and the series converges if

$$
\left|\frac{a_{0}}{a_{1}}\right|^{2}<\min \left(\frac{1}{n e \sum_{j=2}^{n}\left|\frac{a_{j}}{a_{0}}\right|}, 1\right) .
$$


Proof. Let $r=-a_{0} / a_{1}, M=\sum_{j=2}^{n}\left|\frac{a_{j}}{a_{0}}\right|, N_{v}=\sum_{j=2}^{n} v_{j}, K_{v}=\sum_{j=2}^{n} j v_{j}$, and put

$$
Z(\gamma)=\frac{\tilde{X}}{\gamma r^{\gamma}}=\sum_{v_{2}, \ldots, v_{n} \geq 0} \frac{\Gamma\left(\gamma+K_{v}\right) r^{K_{v}}}{\Gamma\left(1+\gamma+K_{v}-N_{v}\right)} \prod_{j=2}^{n} \frac{a_{j}^{v_{j}}}{a_{0}^{v_{j}} v_{j} !}
$$

where $\tilde{X}$ is the right hand side of (17). Let $0 \leq i \leq \gamma \leq i+1$ and $|r|<1$ and observe that $2 N_{v} \leq K_{v} \leq n N_{v}$. We then have

$$
\begin{aligned}
|Z(\gamma)| & \leq \sum_{v_{2}, \ldots, v_{n} \geq 0} \frac{\left(i+K_{v}\right) !|r|^{K_{v}}}{\left(i+K_{v}-N_{v}\right) !} \prod_{j=2}^{n} \frac{\left|\frac{a_{j}}{a_{0}}\right|^{v_{j}}}{v_{j} !} \\
& \leq \sum_{v_{2}, \ldots, v_{n} \geq 0}\left(i+n N_{v}\right)^{N_{v}}|r|^{2 N_{v}} \prod_{j=2}^{n} \frac{\left|\frac{a_{j}}{a_{0}}\right|^{v_{j}}}{v_{j} !} \\
& =\sum_{N=0}^{\infty} \frac{(i+n N)^{N}|r|^{2 N}}{N !} \sum_{v_{2}+\cdots+v_{n}=N} N ! \prod_{j=2}^{n} \frac{\left|\frac{a_{j}}{a_{0}}\right|^{v_{j}}}{v_{j} !} \\
& =\sum_{N=0}^{\infty} \frac{(i+n N)^{N}|r|^{2 N} M^{N}}{N !}=\sum_{N=0}^{\infty} b_{N} .
\end{aligned}
$$

Furthermore, $b_{N+1} / b_{N} \rightarrow e n M|r|^{2}$ as $N \rightarrow \infty$, so $Z(\gamma)$ converges uniformly on $\gamma \in[i, i+1]$ if $|r|<\frac{1}{\sqrt{n M e}}$ and $|r|<1$. From the uniform convergence it follows that $Z(\gamma) \in C\left(\mathbb{R}^{+}\right)$, since $\frac{\Gamma\left(\gamma+K_{v}\right)}{\Gamma\left(1+\gamma+K_{v}-N_{v}\right)} \in C\left(\mathbb{R}^{+}\right)$and $i \in \mathbb{N}$ is arbitrary. $X^{\gamma}$ is continuous with respect to $\gamma$, and since $X^{\gamma}$ equals $\tilde{X}=\gamma r^{\gamma} Z(\gamma)$ on a dense subset of $\mathbb{R}^{+}$, the theorem follows.

Remark. When $i=0$, we should have $0<\gamma \leq 1$, and we also note that the estimates in the proof fail for one term. This does not affect the conclusion.

\section{$6 \quad$ Examples}

In this section we give examples of how to find expressions for $\tilde{\alpha}$ given only the multipole moments. We start with the pure $2^{n}$-poles which include the Schwarzschild solution and the gravitational dipole of [7]. We then give $\tilde{\alpha}$ for the monopole $-2^{n}$-poles, which in particular include the monopole-quadropole solution of Hernández-Pastora and Martín, 9]

\subsection{Pure $2^{n}$-pole}

For the pure $2^{n}$-pole we have $y(\rho)=q \frac{\rho^{n}}{n !}$, so that equation (9) reduces to

$$
\frac{(1+2 n) q^{2}}{n !(n+1) !} \rho^{2 n+2}-\frac{\rho}{r}+1=0 .
$$

The solution $\rho(r)$ with the appropriate asymptotics is given by (16), while $Y(r)=\frac{q \rho^{n+1 / 2}}{\sqrt{r} n !}$ is given by (17). The resulting power series expression is

$$
Y(r)=\frac{r^{n} q}{n !} \sum_{i=0}^{\infty} \frac{(2 n+1) \Gamma(2(n+1) i+n+1 / 2) r^{2(n+1) i} c^{i}}{2 \Gamma((2 n+1) i+n+3 / 2) i !}
$$


where $c=\frac{(1+2 n) q^{2}}{n !(n+1) !}$. In terms of $\tilde{\alpha}$ we get

$$
\tilde{\alpha}(r, \theta)=\frac{(2 n+1) q}{n !} \sum_{i=0}^{\infty} \frac{(2(n+1) i+n) ! \sqrt{\pi} c^{i} r^{2(n+1) i+n} P_{2(n+1) i+n}(\cos \theta)}{\Gamma((2 n+1) i+n+3 / 2) i ! 2^{2(n+1) i+n+1}} .
$$

This simple form makes it possible to express $\tilde{\alpha}(r, 0)$ in terms of a hypergeometric function:

$\tilde{\alpha}(r, 0)=\frac{q r^{n}}{(2 n-1) ! !} 2 n+2 F_{2 n+1}\left(\left[\frac{n+1}{2 n+2}, \frac{n+2}{2 n+2}, \ldots, \frac{3 n+2}{2 n+2}\right],\left[\frac{2 n+3}{4 n+2}, \frac{2 n+3+2}{4 n+2}, \ldots, \frac{6 n+3}{4 n+2}\right], z\right)$,

where $z=\frac{(n+1)^{2 n+1} q^{2}}{(2 n+1)^{2 n} n !^{2}} r^{2 n+2}$. In particular, (22) with $n=0$ with $q=m$ gives

$$
\tilde{\alpha}(r, 0)=m_{2} F_{1}\left(\left[\frac{1}{2}, \frac{2}{2}\right],\left[\frac{3}{2}\right], m^{2} r^{2}\right)=\frac{1}{2} \log \frac{1+m r}{1-m r},
$$

i.e., the Schwarzschild solution. $n=1$ gives

$$
\tilde{\alpha}(r, 0)=q r_{4} F_{3}\left(\left[\frac{2}{4}, \frac{3}{4}, \frac{4}{4}, \frac{5}{4}\right],\left[\frac{5}{6}, \frac{7}{6}, \frac{9}{6}\right], \frac{8}{9} m^{2} r^{4}\right),
$$

i.e., the gravitational dipole of [7].

\subsection{Monopole - $2^{n}$-pole}

Space-times with given monopole-moment $m$ and $2^{n}$-pole-moment $q$ have direct physical interpretations. They describe pure relativistic $2^{n}$-pole corrections to the Schwarzschild solution. Of particular interest is the monopole-quadropole solution, since it in a sense ${ }^{4}$ represents the lowest order correction. This case is considered in section (6.3). In this section we derive the the metric for the general monopole - $2^{n}$-pole.

A space-time with monopole $m$ and $2^{n}$-pole $q$ is given by $y(\rho)=m+\frac{q}{n !} \rho^{n}$. Equation (9) then reduces to

$$
c_{1} \hat{q}^{2} \hat{\rho}^{2 n+2}+c_{2} \hat{q} \hat{\rho}^{n+2}+\hat{\rho}^{2}-\frac{\hat{\rho}}{\hat{r}}+1=0,
$$

where $c_{1}=\frac{2 n+1}{(n+1) ! n !}, c_{2}=\frac{8 n+4}{(n+2) !}, \hat{\rho}=m \rho, \hat{q}=m^{-(n+1)} q$, and $\hat{r}=m r$. The solution with the appropriate asymptotics is then given by (16), while the $\gamma$ power is given by (17). The solutions simplifies to

$$
\begin{aligned}
\hat{\rho} & =\hat{r} \sum_{i, j, k \geq 0} \frac{\Gamma\left(F_{n i j k}+1\right) \hat{q}^{j+2 k} c_{2}^{j} c_{1}^{k}}{i ! j ! k ! \Gamma\left(G_{n i j k}+1\right)} \hat{r}^{F_{n i j k}}, \\
\hat{\rho}^{\gamma} & =\hat{r}^{\gamma} \gamma \sum_{i, j, k \geq 0} \frac{\Gamma\left(F_{n i j k}+\gamma\right) \hat{q}^{j+2 k} c_{2}^{j} c_{1}^{k}}{i ! j ! k ! \Gamma\left(G_{n i j k}+\gamma\right)} \hat{r}^{F_{n i j k}},
\end{aligned}
$$

where we have used the index functions $F_{n i j k}=2 i+(n+2) j+(2 n+2) k$, $G_{n i j k}=i+(n+1) j+(2 n+1) k+1$. The function $Y$ is then given by

$$
\begin{aligned}
Y & =\sqrt{\frac{\rho}{r}} y(\rho)=\frac{m \hat{\rho}^{1 / 2}}{\hat{r}^{1 / 2}}+\frac{m \hat{q} \hat{\rho}^{n+1 / 2}}{n ! \hat{r}^{1 / 2}} \\
& =\sum_{i, j, k \geq 0} \frac{m \hat{q}^{j+2 k} c_{2}^{j} c_{1}^{k} \hat{r}^{F_{n i j k}}}{i ! j ! k !}\left(\frac{\Gamma\left(F_{n i j k}+\frac{1}{2}\right)}{2 \Gamma\left(G_{n i j k}+\frac{1}{2}\right)}+\frac{(2 n+1) \hat{q} \hat{r}^{n} \Gamma\left(F_{n i j k}+n+\frac{1}{2}\right)}{2 \Gamma\left(G_{n i j k}+n+\frac{1}{2}\right) n !}\right) .
\end{aligned}
$$

\footnotetext{
${ }^{4}$ If the mass is non-zero, the dipole moment can always be put to zero.
} 
After some simplifications we get the following expression for $\tilde{\alpha}$ :

$$
\begin{aligned}
\tilde{\alpha}(r, \theta)= & \sum_{i, j, k \geq 0} \frac{m \hat{q}^{j+2 k} c_{2}^{j} c_{1}^{k} \hat{r}^{F_{n i j k}}}{i ! j ! k ! 2^{i+j+k}}\left(\frac{F_{n i j k} ! P_{F_{n i j k}}(\cos \theta)}{\left(2 G_{n i j k}-1\right) ! !}\right. \\
& \left.+\frac{(2 n+1) \hat{q} \hat{r}^{n}\left(F_{n i j k}+n\right) ! P_{F_{n i j k}+n}(\cos \theta)}{n !\left(2 G_{n i j k}+2 n-1\right) ! !}\right)
\end{aligned}
$$

so that the corresponding space-time is now explicitly given.

\subsection{The monopole-quadropole conjecture of Hernández- Pastora and Martín}

The monopole-quadrupole (static axisymmetric) solution, i.e., the monopole- $2^{2}$ pole has been studied by Hernández-Pastora and Martín. In [9] they conjectured an expression for this solution with mass parameter $m$ and quadrupole moment $q$. With a minor change of conventions ( $\operatorname{sign}$ and $3 q \rightarrow q$ ), the conjectured solution is

$$
\tilde{\alpha}(r, 0)=\sum_{i, j, k} \frac{m^{2 i+1} r^{2 i} \hat{q}^{j} i !(2 i-1) ! ! 3^{-k} 5^{k}(4 j-3 k+1)}{(2 i+2 j+1) ! !(i-j-k) !(j-k) !(2 k-j+1) !} .
$$

Here the summation is taken over all integers $i, j, k$ subject to the conditions $i \geq 0, i-j-k \geq 0, j-k \geq 0,2 k-j+1 \geq 0$. Adopting the convention that $1 / i !=0$ if $i$ is a negative integer, the summation is taken over all integers $i, j, k$ subject to $i \geq 0$. Also, $(-1) ! !=1$. We prove this conjecture by rewriting (23). From (23) we get

$$
\tilde{\alpha}(r, 0)=\sum_{i, j, k} \frac{m \hat{q}^{j+2 k} c_{2}^{j} c_{1}^{k} \hat{r}^{2 i+4 j+6 k}}{i ! j ! k ! 2^{i+j+k}}\left(\frac{(2 i+4 j+6 k) !}{(2 i+6 j+10 k+1) ! !}+\frac{5 \hat{\hat{r}^{2}(2 i+4 j+6 k+2) !}}{2(2 i+6 j+10 k+5) ! !}\right),
$$

where $c_{2}=5 / 6, c_{1}=5 / 12$ and where by the convention the summation is effectively taken over all nonnegative integers. In the first term, let $i \rightarrow i-2 j-3 k$ and in the second $i \rightarrow i-2 j-3 k-1$. After this change we let $j \rightarrow j-2 k$ in the first term and $j \rightarrow j-2 k-1$ in the second, and obtain

$$
\tilde{\alpha}(r, 0)=\sum_{i, j, k} \frac{m \hat{q}^{j} c_{2}^{j-2 k} c_{1}^{k} \hat{r}^{2 i}(2 i) !}{(2 i+2 j+1) ! ! k ! 2^{i-j}}\left(\frac{1}{(i-2 j+k) !(j-2 k) !}+\frac{5}{2 c_{2}(i-2 j+k+1) !(j-2 k-1) !}\right) .
$$

Next, we let $k \rightarrow k-1$ in the second term and obtain

$$
\tilde{\alpha}(r, 0)=\sum_{i, j, k} \frac{m \hat{q}^{j} 5^{j-k} 3^{k-j} \hat{r}^{2 i}(2 i) !}{(2 i+2 j+1) ! ! k ! 2^{i}(i-2 j+k) !}\left(\frac{1}{(j-2 k) !}+\frac{5 k}{(j-2 k+1) !}\right) .
$$

Using $(2 i) ! / 2^{i}=(2 i-1) ! ! i !$ and rewriting the first term we get

$$
\tilde{\alpha}(r, 0)=\sum_{i, j, k} \frac{m \hat{r}^{2 i} \hat{q}^{j} 5^{j-k} 3^{k-j} i !(2 i-1) ! !(j+3 k+1)}{(2 i+2 j+1) ! ! k !(i-2 j+k) !(j-2 k+1) !} .
$$

After the change $k \rightarrow j-k$ we finally get (24). 


\section{Conclusions and discussion}

We have investigated the Geroch moments which are defined through the recursion (11). In [7, it was shown how this recursion in the axisymmetric case can be replaced by a much simpler recursion involving only scalar valued functions, and how all moments can be captured in one single function.

In this work, we have showed how these results can be used to construct static axisymmetric space-times with prescribed moments. In the case of finite number of non-zero moments, we have shown how to obtain the metric of the corresponding space-time explicitly in terms of power series. In addition, using (23) we were able to confirm the monopole-quadropole conjecture by HernándezPastora and Martín [9]. In the general axisymmetric case, where we have the implicit relation (9), we were able to confirm a special case of a conjecture due to Geroch [5].

For further studies of the solutions presented here, integral representations or other analytical expressions would be very useful. In particular, this could give further insight for the pure $2^{n}$-poles (21).

It is natural to try and extend the framework presented here to more general cases, like static non-axisymmetric or stationary space-times. For instance, it would be instructive to calculate the moments of the Kerr solution which are stated in 8 .

It is also interesting to note that one can define 'addition' of space-times, by simply adding their Geroch moments. For instance, we can 'add' two Schwarzschild solutions using (8) with different displacements. Displacing the solutions with $\kappa^{\prime}(0)= \pm 1$ and choosing $m=1$, addition of the Geroch-moments gives

$y(\rho)=\frac{1}{\sqrt{1+\rho}}+\frac{1}{\sqrt{1-\rho}}$ and by (9) we get

$$
-4 \sqrt{1-\rho^{2}}+3+\frac{2}{1-\rho^{2}}-\frac{\rho}{r}=0 .
$$

This equation is algebraic in terms of $z=\left(1-\rho^{2}\right)^{-1 / 2}$, and can therefore be studied with the tools provided here. Thus, it may be possible to investigate the physical relevance of such an addition.

\section{References}

[1] Beig, R., Simon, W., Proof of a Multipole Conjecture due to Geroch, Commun. Math. Phys., 78, 75 (1980).

[2] Beig, R., The Multipole Expansion in General Relativity, Acta Physica Austriaca, 53, 249 (1981).

[3] Fodor, G., Hoenselaers, C., Perjés, Z., Multipole moments of axisymmetric system in relativity, J. Math. Phys., 30, 2252 (1989).

[4] Gel'fand, I.M., Zelvinsky, A.V., Kapranov, M.M., Hypergeometric functions and toral manifolds, Functional Anal. Appl., 23, 94 (1989).

[5] Geroch, R., Multipole Moments. II. Curved Space, J. Math. Phys., 11, 2580 (1970). 
[6] Gürsel, Y. Multipole moments for stationary systems - The equivalence of the Geroch Hansen formulation and the Thorne formulation, Gen. Rel. Grav. 15737 (1983).

[7] Herberthson, M., The gravitational dipole and explicit multipole moments of static axisymmetric space-times, Class. Quantum Grav. 21, 5121 (2004).

[8] Hansen, R.O., Multipole moments of stationary space-times, J. Math. Phys., 15, 46 (1974).

[9] Hernández-Pastora, J.L., Martín, J., Monopole-Quadropole Static Axisymmetric Solutions of Einstein Field Equations, Gen. Rel. Grav., 26, 877 (1994).

[10] Hernández-Pastora, J.L., Martín, J., Ruiz,E., Approaches to MonopoleDynamic Dipole Vacuum Solution Concerning the Structure of its Ernst Potential on the Symmetry Axis, Gen. Rel. Grav., 30, 999 (1998).

[11] Quevedo, H., Multipole Moments in General Relativity - Static and Stationary Vacuum Solutions, Fortschritte der physik, 38, 733 (1990).

[12] Rudin, W., Real and Complex Analysis, second edition, McGraw-Hill, New York 1986.

[13] Sturmfels, B., Solving algebraic equations in terms of $\mathcal{A}$-hypergeometric series, Discrete Mathematics, 210, 171 (2000).

[14] Thorne, K.S., Multipole expansions of gravitational radiation, Rev. Mod. Phys., 52, 299 (1980).

[15] Weyl, H., Zur Gravitationstheorie, Ann. Phys., 54, 117 (1917). 